\title{
Warum die Atomenergie das Zivilrecht nicht kalt läßt
}

$I$.

Das Amtsgericht Stuttgart hat in einer weithin beachteten und überwiegend geschmähten Entscheidung ${ }^{1}$ die Klage der Technischen Werke der Stadt Stuttgart (TWS) gegen eine Reihe von Stromabnehmern mals zur Zeit unbegründet abgewiesen «, die $10 \%$ der laufenden Stromrechnungen nicht an die TWS zahlten, sondern auf einem Sperrkonto festlegten, "bis die Klägerin von der Errichtung und dem Betrieb von Kernkraftwerken Abstand nimmt«. Im Gegensatz zu anderen Entscheidungen mit vergleichbarem Sachverhalt ${ }^{2}$ geht das AG Stuttgart davon aus, daß die Beklagten ein Zurückbehaltungsrecht gemäß $\$ 273$ BGB geltend machen können, das aus dem Verstoß der Klägerin gegen vertragliche Nebenpflichten resultiere. Dieser Verstoß bestehe darin, daß sie "zwar die an sich ungefährliche Ware Strom" liefere, diesen aber in einer Weise herstelle, "die den Beklagten und mit ihm die Allgemeinheit gefährdet «. Die erforderlichen (Teil-)Betriebsgenehmigungen dazu seien der Klägerin zwar erteilt worden, diese seien aber angefochten, nicht rechtsbeständig, also "nur eine formale Rechtsposition «, die lediglich "bis zu einem gewissen Grade« entlaste. Darüber hinaus seien seit der Erteilung der Betriebsgenehmigung schwerwiegende faktische Veränderungen eingetreten. Zum einen sei seit dem Reaktorunfall in Harrisburg "offenkundig..., daß die Atomtechnik nicht beherrscht wird und daß es gegen ihre Gefahren keinen Schutz gibt «. Zum anderen sei seit der Entscheidung, die Wiederaufbereitungsanlage in Gorleben nicht zu bauen, "das Problem der Entsorgung ungelöst, das aber nach dem Atomgesetz die Voraussetzung dafür ist, daß Kernkraftwerke rechtmäßig betrieben werden dürfen«.

II.

Das Urteil bewegt sich zweifellos nicht in den sicheren Bahnen zivilistischer Dogmatik. Daß dies aus der ungewöhnlichen Tragweite des Konfliktes selbst resultiert, gibt das AG Hamburg noch in seiner Kritik zu erkennen, wenn es ausführt, daß die Bewertung der Kernenergierisiken nicht Aufgabe eines Zivilgerichtes sei, "solange die Verwaltungsgerichtsbarkeit funktioniert «. ${ }^{3}$

Ohne dagegen auf die schwerwiegende Bedeutung einzugehen, hat Lüke eine vernichtende Anmerkung verfaßt, wobei er allerdings eher nach dem Grundsatz ,fortiter in modo, suaviter in re verfahren ist: Er folgt der sich rasch ausbreitenden Mode, Gegner in juristischen und politischen Auseinandersetzungen, die vom eigenen, autoritären Vorurteil abweichen, mit dem Makel der Faschismus-Affinität

\footnotetext{
I AG Stuttgart vom 20. Juli 1979 - $7 \mathrm{C}_{4630 / 79}$ - teilweise abgedruckt in NJW 1979, 2047 mit Anm. von Lüke NJW 1979, 2049 sowie in ]Z 1979, 809 mit Anm. von E. Schwerdtner. Es ist zu begrüßen, daß das BVerfG angesichts der unmäßigen Urteilskritik seine Kompetenzanmaßungen nicht noch weiter getrieben hat: es hat die Annahme der Verfassungsbeschwerde gegen das rechtskräftige Urteil abgelehnt und damit darauf verzichtet, neben dem Super-Parlament nun auch noch Super-BGH zu werden; vgl. I BvR $834 / 79$, zit. nach FAZ vom 19. 1. 1980, S. 7. Die folgenden Zitate aus der Entscheidung entnehme ich einer Urteils-Abschrift.

2 Vgl. etwa AG Hamburg vom 18.9. 1979-21 b C 920/78-NJW 1979, 2315; AG Hamburg vom 23. 8. 1978 - ${ }_{10} \mathrm{C}_{414} / 78$.

3 NJW 1979, 2316 - Hervorhebung von mir, R. K.

4 Lüke, a. a. O., S. 2050.
} 
zu belegen ${ }^{4}$, wie es vor ihm bereits Ärzte-Funktionäre` und Bischöfe ${ }^{6}$ in ihren Diffamierungs-Kampagnen gegen jede Reform der strafrechtlichen Verfolgung von Schwangerschaftsabrüchen getan haben. Diese Dimension der Kritik ist "peinlich « - um es mit einem Begriff Lükes zu sagen -, da sie nicht nur zu deutlich belegt, wie gering der historische Bildungsstand über den Faschismus bei manchen Ärzten, Bischöfen und Professoren ist ${ }^{7}$, sondern auch, wie einäugig die "politische Funktion der Privatrechtsinstitute ${ }^{8}$ betrachtet wird: Lüke hat es nicht "peinlich " gefunden (oder wenigstens peinliche Berührung nicht offenbart), als das LG Berlin ein korrekt abgeliefertes Werk gleichwohl als Nichterfüllung qualifizierte, weil der Werkherste]ler privat eine mißliebige politische Uberzeugung pflegte ${ }^{9}$, er hat sich nicht gegen die offen politisch-disziplinierende Funktion der Demonstrations-Urteile des BGH in Zivilsachen ausgesprochen ${ }^{10}$, auch blieb sein Protest gegen die unterschiedliche Behandlung von Herrenreitern und mißliebigen Schriftstellern bei Schmerzensgeldansprüchen wegen Ehrabschneidung ${ }^{\text {I }}$ aus.

Alle hier angegebenen Beispiele belegen die Selbstverständlichkeit, daß dem Zivilrecht - und nicht nur in diesen Fällen - eine politische Funktion zukommt, was nun allerdings weder mit Freirechts-Schule noch mit sunbegrenzter Auslegung، (Rüthers) gleichgesetzt werden darf: gerade auch der streng hermeneutische Positivismus ist politisch, wiederum ohne ihn leicht in >linke oder 'rechte Schubladen einordnen zu können. ${ }^{\prime 2}$ Es kann deshalb einem Urteil nicht vorgeworfen werden, daß es in einem politischen Konflikt politisch argumentiert: vielmehr ist dies gerade ein Vorzug gegenüber anderen Urteilen, die ebenfalls politische Konflikte lösen, ohne die politischen $>$ Vorverständnisse، (Esser) preiszugeben.

Ein deutliches Beispiel für versteckte politische Argumentation liefert im übrigen Lüke selbst: In seinem Bemühen, die Rechtsmittelfähigkeit herzustellen, will er den Beschwerdewert nach dem geltend gemachten Gegenanspruch bemessen, obwohl dieser nur einredeweise geltend gemacht wurde, und obwohl $\$$ s I a ZPO eindeutig vom Gegenteil ausgeht; ${ }^{13}$ er wendet das Prinzip der Meistbegünstigung an, indem er seine Auffassung von einer korrekten Prozeßführung als Alternative einführt und außer Acht läßt, daß das Gericht ein Urteil sprechen konnte und wollte; ${ }^{14}$ er schließt endlich - mit drohendem Unterton - von den seiner Ansicht nach vorliegenden Rechtsfehlern auf Befangenheit der Richterin, ein Verfahren, durch das jede Aufhebung eines Urteils durch höhere Instanzen als Beweis für die Befangenheit der Richter der Vorinstanz gelten müßte. "s

Da die Aussage von der politischen Prägung der juristischen Arbeit sofort interessiert und gezielt mißverstanden wird, ist sie zu ergänzen durch die weitere, daß damit wiederum nicht die Bindung ans Gesetz geleugnet wird: geleugnet wird lediglich, daß Interpretationen des Gesetzes frei von politischen Erwägungen sind.

s Vgl. etwa die Ausführungen des Präsidenten der Bundesärztekammer Dr. Karsten Vilmar, in: Deutsches Ärzteblatt 31/1979, 1985 ff.

$6 \mathrm{Vgl}$. etwa den Hirtenbrief der deurschen Bischöfe, Dem Leben dienen, I979-dazu den Artikel von A. Lorenzer in diesem Heft.

7 Ihnen sei die Leknüre des Sonderbandes der KJ >Der Unrechtsstaar` ( 1979 ) ans Herz gelegt.

8 Ballerstedt, Zur zivilrechtlichen Haftung von Demonstrationsschäden, JZ 1973, ros.

9 Dazu Eike Schmidt, DuR 1977 , ss ff.

Io Vgl. etwa BGH JZ 1973, 123 und den in Fn. 8 zitierten Aufsatz von Ballerstedt.

I I Vgl. dazu Nettelbeck, KJ 1978, I 35 ff.

I2 Dies verkennt etwa H. Klenner, Rechtsleere - Verurteilung der Reinen Rechtslehre, 1972, in seiner hilflosen Kritik an Kelsens Reiner Rechtslehre.

1 3 Vgl. etwa Grunsky in Stein-Jonas, Kommentar zur ZPO, 19. Aufl. 1964-1972, Anm. 2 zu $\ 546$.

14 Vgl. etwa Grunsky, a. a. O., Einl. III vor $\$$ \$1 .

Is Vgl. zur Befangenheitsproblematik etwa Baumbach-Lauterbach, ZPO, 37. Aufl. 1979, Anm. 2 zu $\ 42$. 
Auf der Grundlage dieser Uberlegungen lautet die Ausgangsfrage nicht, ob ein Urteil von politischen Erwägungen gereinigt ist - dies führt ja nur dazu, daß etwas unter den Teppich gekehrt wird -, sie lautet vielmehr, ob die politischen Erwägungen die disziplinierende Grenze der Gesetzes- und Interpretationsbindung überschritten haben. In diesem Zusammenhang ist es zweitrangig, ob ein Zurückbehaltungsrecht aus $₫ 320$ BGB oder $\ 273$ BGB konstruiert wird ${ }^{16}$ : Im Gegensatz zur Ansicht Lükes ist die Anwendung des $\$ 273$ bei gegenseitigen Verträgen nicht durch $\$ 320$ vollständig verdrängt, sondern behält gerade in Fällen wie dem vorliegenden Bedeutung, wo nicht die Haupt-Gegenleistung verweigert wird: die Lieferung von Strom, sondern wo die Beklagten eine Unterlassung verlangen, nämlich das Absehen der Klägerin von ihren Plänen, die Stromversorgung auch der Beklagten durch Kernenergie sicherzustellen. Daß eine Leistungsverpflichtung im Unterlassen einer Handlung liegen kann, steht außer Streit, und ich tendiere in diesem Zusammenhang eher zu der Ansicht, die Grundlage in $\$ 273$ als in $\$ 320$ zu suchen, eben weil es sich nicht um die engere synallagmatische Beziehung handelt. ${ }^{17}$

Korrekt hingegen ist Lükes Kritik an der Tenorierung des Urteils: das Gericht hätte entweder die Klage als unbegründet abweisen müssen, dann aber nicht mit $\$ 273$ oder $\$ 320$ argumentieren dürfen, sondern mit $\ 242$ argumentieren müssen - wobei es sich ohnehin bei $\$ 273$ und $\$ 320$ nur um eine »besondere Ausformung des Prinzips von Treu und Glauben « handelt ${ }^{18}$-, oder es hätte - auf der Grundlage der eigenen Entscheidung - Zug um Zug gegen Unterlassen des weiteren Betreibens der Kernenergiepläne bis zur Lösung der Abfallbeseitigungsprobleme und Sicherheitsrisiken verurteilen müssen.

Der Kern des Problems liegt jedoch an anderer Stelle: es geht darum, ob - wie es Ballerstedt in anderem Zusammenhang polemisch gefragt hat - "die Rechtsgüter anderer ... um irgendwelcher Freiheitsideale willen aufgeopfert werden dürfen «. ${ }^{19}$ Diese Frage hat Ballerstedt im Zusammenhang des Demonstrations-Zivil-Rechts vehement verneint - in einer entschieden angreifbaren Abwägung zwischen der Freiheit zu unternehmerischer Betätigung und Freiheit der politischen Meinungsäußerung in einer bestimmten historischen Situation. Im Bereich der Kernenergie stellt sich die Frage dramatischer: Die Einschätzung der Gefahren der Kernenergie durch das AG Stuttgart ist keine abwegige private Spekulation, sondern wissenschaftlich gut belegt und auf dieser Ebene ernsthaft diskutiert. Auf dieser - wissenschaftlich vertretbaren - Grundlage stellt sich für das Gericht die Alternative, die Beklagten mit einem die Klage stattgebenden Urteil zu verpflichten, Forschung an und Produktion von Energie-Trägern (mitzu-)finanzieren, die das Leben der Beklagten selbst bedrohen. Diese Dimension sprengt die Regeln des klassischen Privatrechts. Der Hinweis auf die Privatautonomie und auf die Möglichkeit der Beklagten, dann eben keinen Vertrag mit der Klägerin zu schließen, d. h. keinen Strom zu beziehen und ein durchschnittliches Leben aufzugeben, ist mit dem Unterton des »Zurück in die Steinzeit« ebenso hämisch wie hilflos und erinnert an das alte "Geht doch in die DDR, wenn es Euch hier nicht paßt!*. Gleichzeitig widerspricht es dem Kontrahierungszwang, dem die Energieversorgungsunternehmen unterliegen. Der Verallgemeinerung des Urteils zu der Konsequenz, daß nun in Zukunft jeder seine

16 Darauf legt Lüke besonderen Wert, a. a. O., S. 2049.

I7 Vgl. etwa Keller in Münchener Kommentar, BGB, Schuldrecht Allgemeiner Teil, 1979, \273 Rdnr. 77.

18 Keiler, a. a. O., Rdnr. 1.

rg Ballerstedt, a. a. O., S. 106. 
Vertragserfüllung mit dem Hinweis auf politisch mißliebige (Investitions-)Politik verweigern könne, (bisher hat nur das o. a. Urteil des LG Berlin die Möglichkeit solcher Erwägungen in Zivilprozessen angedeutet, um eine »linke « Úberzeugung zu diskriminieren), geht es wie vielen auf die Spitze getriebenen Vergleichen: sie werden in der Zuspitzung absurd und verlieren ihre Aussagekraft. Das Spezifikum der Energieversorgung liegt in der staatlich geschützten Monopolstellung des Vertragspartners. Aus diesem Grunde ließe sich eine inhaltliche Parallele eher zu der während des Vietnam-Krieges in den USA geübten Praxis ziehen, Bundessteuern zu einem Teil auf Sonderkonten zu zahlen und zu sperren, um die Finanzierung des Krieges zu unterlaufen. Obwohl es hier um öffentlich-rechtliche Steuerzahlung, dort um die Erfüllung von Vertragspflichten geht, läßt sich die Parallelität nicht von der Hand weisen, da Verträge mit monopolistischen Versorgungsträgern trotz der privatunternehmerischen Form wenig mit dem klassischen Vertragsmodell gemein haben.

Das moderne Privat- und Wirtschaftsrecht erkennt derartige Veränderungen des Vertragsmodells - neben dem hier bestehenden Kontrahierungszwang selbst - in verschiedenen Beziehungen längst an:

- Mit der spätestens seit der 'Parkplatz<-Entscheidung ${ }^{20}$ des BGH durchgesetzten Konstruktion des faktischen Vertrages ist das Moment der Willensentschließung beim Vertragsschluß zugunsten der 'Sozialtypik von Massenverträgen weitgehend zurückgedrängt worden.

- Das AGB-Gesetz hat mit $\ 2$ die Vorstellung vom Vertrag als einer sfrei ausgehandelten Rechten- und Pflichtenordnung< endgültig gesetzlich hinter sich gelassen,

- $\$ 22$ GWB und die diese Bestimmung interpretierende ständige Rechtsprechung des BGH gehen davon aus, daß marktbeherrschende Unternehmen hoheitlich zu (Sukzessiv-)Lieferungen zu normalen Bedingungen wie zur Berechnung von Höchstpreisen verpflichtet werden können. ${ }^{25}$

Diese für die Veränderung des Zivilrechts zeugenden Beispiele ließen sich um solche aus dem Wettbewerbsrecht, dem gewerblichen Rechtsschutz, dem sallgemeinen Zivilrecht ergänzen. Sie könnten als gesetzliche Kehrseite der Praxis marktmächtiger Vertragspartner qualifiziert werden, auf Vertragsbedingungen zu bestehen, die weit in die Geschäftspolitik des anderen Partners eindringen - etwa bei der Vergabe von Krediten durch Großbanken -, ohne daß ein solches Verhalten für zivilrechtlich unzulässig gehalten würde.

Für unseren Zusammenhang sind die Beispiele aus mehreren Gründen relevant: Sie belegen, daß inzwischen weder das Gesetz noch die Rechtsprechung weiterhin davon ausgehen, daß im Vertrag zwei autonome Individuen aufeinandertreffen, die isoliert ihre privaten Interessen durchsetzen, die zu gesellschaftlichen nur mittelbar, durch eine intellektuelle Konstruktion werden: in der Verfolgung der Eigeninteressen - so hieß es - werde die allgemeine Wohlfahrt gefördert, so daß - in Konsequenz - jeder, der sein Recht verfolge, damit das Recht im allgemeinen verteidige. ${ }^{22}$

Wenn ein auf Stromlieferung angewiesener Kunde einem Versorgungsmonopolisten

\footnotetext{
$20 \mathrm{BGHZ} 2 \times, 319$.

21 BGHZ 67, 104; BGHZ 68, 23; weitergehend etwa Reich, Markt und Recht, 1977, S. 202 f.

22 Vgl. statt aller die unverändert schöne juristische Ausarbeitung dieser Gedankenführung bei R. v. Ihering, Der Kampf ums Recht, 4. Aufl. 1874; vgl. auch die sich darauf beziehende Argumentation von Rehbinder/Burgbacher/Knieper, Bürgerklage im Umweltrecht, 1972. Zur staatstheoretischen Dimension vgl. Knieper, Weltmarkt, Wirtschaftsrecht und Nationalstaat, 1976, bes. S. I I ff./236 ff.
} 
gegenübersteht, dann verliert dieses Argument auch noch jeden Rest der Legitimationskraft des Vertragsmodells. Gerade die Verteidiger der 'modernen Industriegesellschaft< müßten sich für dieses offensichtliche Versagen des Marktes bei der Herstellung des sallgemeinen Wohls‘ um Alternativen bemühen, ohne sich schmollend auf die schale Forderung nach mehr Markt und Leistungswettbewerb zurückzuziehen, die den Konzentrationsprozeß von Anfang an als eine Art Hof-Narretei begleitet. Dabei liegt es nahe, auf unmittelbare Berücksichtigung des öffentlichen Wohls zu drängen, wenn die Vermittlung über die Verfolgung des Eigeninteresses an den für Vertragsschlüsse notwendigen Bedingungen scheitert.

Das Verhalten der Beklagten liegt auf dieser Linie: sie verhalten sich privatrechtssystemkonform und - wenn man so will - spät-neo-liberal, da sie einer offenbaren Defizienz des privaten Vertrages, der nach der neoliberalen Theorie seinen Wert geradezu aus dem Fehlen dieser Defizienz erhält, innovativ abhelfen: Nur wer davon ausgeht, daß im Durchschnitt die Verfolgung privater Interessen das allgemeine Wohl fördere, kann ja den Vertrag legitimieren. Wenn sich herausstellt, daß dies in einer bestimmten Konstellation unter keinen Umständen möglich ist, dann ist es aus der allgemeinen Rechtfertigung des Vertragsmodells gerechtfertigt, die Verfolgung des allgemeinen Wohls auch im Rahmen einer Austauschbeziehung auf andere Weise zu betreiben, und dann gehören die vom Gericht gestellten Fragen nach der monopolistischen Angebotsstruktur und nach der lebensbedrohenden Qualität der Forschung zu den zentralen Fragen eines Zivilprozesses, der allerdings mit dem >klassischen، Zivilprozeß ebensowenig zu tun hat wie der Handwerkermeister mit dem Energieversorgungsunternehmen.

Gerade deshalb ist das Verhältnis zwischen Kläger und Beklagten nur unzureichend als Vertragsverhältnis umschrieben. Es ist sehr viel umfassender - und dies im unmittelbaren Wortsinn: das soziale Verhältnis von Angewiesensein auf Stromlieferung und Ausgeliefertsein an die Forschung steht nicht neben dem individual-vertraglichen ,Ware gegen Geld<, sondern jenes umfaßt dieses unentrinnbar. Zu Recht weisen die Beklagten darauf hin, daß sie als Zahlende einen Teil der von ihnen und anderen für lebensbedrohend gehaltenen Technologie mitfinanzieren.

Um dieses allgemeine Problem für das spezielle Vertragsverhältnis operationabel zu machen, greift das Gericht zur Konstruktion der Verletzung einer nebenvertraglichen Pflicht. Damit greift es nicht vollständig daneben, sondern verlängert eine Schiene, an deren Verlegung seit längerer Zeit gearbeitet wird: "Der rasche Wandel und die zunehmende Komplizierung der rechtlichen, wirtschaftlichen, sozialen und technischen Verhältnisse " - so heißt es in der neuesten Auflage des ehrwürdigen Staudinger ${ }^{23}$ - »haben diese Probleme in den Vordergrund gerückt . . . Hinter dieser Entwicklung steht zum einen das Postulat einer stärkeren Verrechtlichung des Gebots der Rücksichtnahme auf den Vertragspartner."Die auf diese Weise sozialpolitisch begründeten Nebenpflichten sollen das Interesse, "vor Schäden an Gesundheit und Eigentum bewahrt zu bleiben «, schützen, sie sollen Risiken mindern, sie können eine Pflicht zur Mitwirkung bei behördlichen Auflagen begründen, sie können weitgehende Untersuchungs- und Prüfungspflichten zum Inhalt haben. ${ }^{24}$ Die Entscheidung des AG Stuttgart geht über diese Beispiele insoweit hinaus, als diese sich wesentlich auf den Kaufgegenstand selbst beziehen, die Modalitäten der Herstellung dagegen in der Verkäufer-Disposition belassen. Dieser Schritt wiegt schwer. Entsprechend schwer haben die Kriterien zu wiegen, die ihn rechtfertigen können. Das Gericht findet sie in der Monopolstellung der Klägerin und der 
Lebensbedrohung der Beklagten sowie weiter Bevölkerungskreise. Solche Argumente sind einer ernsthafteren Diskussion würdig, als sie bisher geführt wurde: Die Atomenergie kann das Zivilrecht nicht kalt lassen. Dabei wird nicht bestritten, daß sie ihren Eingang ins Vertragsrecht mit Hilfe einer dogmatischen Krücke findet, bestritten wird lediglich, daß es sich um die erste Krücke handelt, die in eine ansonsten reibungslos als Textinterpretation von Normen funktionierende juristische Kunstlehre eingreift: die Figur der positiven Vertragsverletzung selbst, aber auch Legionen anderer Krücken - vom Vertrauensprinzip über den faktischen Vertrag, vom normativen Schadensbegriff zum bereicherungsrechtlichen Leistungsbegriff, um willkürlich einige Beispiele zu nennen - bilden einen Teil des Zivilrechts, geschaffen, um als unangemessen erscheinende Lösungen korrigieren zu können und insofern notwendiger Bestand der Dogmatik. Wenn in einer dramatischen sozialen Lage eine neue Krücke geschnitzt wird, so sollte nicht überraschen, daß Schnitzfehler auftauchen, sondern der Versuch gemacht werden, zu einem tragfähigen Konsens zu kommen.

Rolf Knieper

\section{Ehrverletzung durch neonazistische Propaganda}

\section{Zum Urteil des Bundesgerichtshofs vom 18. 9. $1979^{\text {\% }}$}

Dieses Urteil des BGH, welches in der bundesrepublikanischen Offentlichkeit breite Beachtung gefunden hat, setzt den juristischen Schlußpunkt unter ein Verfahren, welches gleichsam als Test auf Entwicklungsstand und Form der "Bewältigung der Vergangenheit « angesehen werden kann. Der Kläger, Student aus Mainz, wandte sich an das dortige Landgericht um gerichtlichen Beistand gegen die neonazistische Propaganda des ebenfalls in Mainz ansässigen Beklagten. Dieser hatte an der Einfriedungsmauer seines Anwesens eine Plakatwand angebracht, auf der er Publikationen der "Aktion Widerstand " sowie rechtsradikaler Publizisten und Verlage öffentlich ausstellte. Da der Kläger jüdischer Abstammung ist - sein Großvater wurde in Auschwitz ermordet - sah er sich insbesondere durch ein Flugblatt des Beklagten in seiner Ehre verletzt, in dem dieser die Ermordung von Millionen Juden im Dritten Reich als zionistischen Schwindel bezeichnete und von der Lüge von den sechs Millionen vergasten Juden sprach. Er verklagte daher den Beklagten auf Unterlassung der beanstandeten Äußerungen.

Nachdem das LG Mainz (Urt. v. 6. 4. I977, KJ I978, S. I 89 ff.) wie bereits im einstweiligen Verfügungsverfahren dem Anspruch des Klägers auch im Hauptverfahren stattgegeben hatte, verneinte das OLG Koblenz (Urt. v. 2. 5. 1978, KJ 1979, S. $193 \mathrm{ff}$., mit Anm. von mir, T. B.) auf die Berufung des Beklagten hin einen Unterlassungsanspruch des Klägers. Es versachlichte die Äußerung des Beklagten zu einer (zwar unrichtigen und tendenziösen) Auffassung über andere Ansichten zur nationalsozialistischen Judenvernichtung, durch die der Kläger in seiner Ehre nicht betroffen werde, weil er weder selbst (vielmehr »die Zionisten «) als Urheber dieser »Lüge « bezeichnet werde noch selbst Jude und als solcher von den Nationalsozialisten verfolgt worden sei.

* JZ 79/8 I f.; im folgenden zitiert nach dem hektographierten Urteilstext. 